\title{
REFLECTION OF METASTABLE HELIUM ATOMS ON A MOLYBDENUM /110/ SURFACE EFFECT OF OXYGEN ADSORPTION
}

\author{
C. BOIZIAU, R. NUVOLONE and J. ROUSSEL
}

Service de Physique Atomique

Centre d'Etudes Nucléaires de Saclay,

BP No 2, 91190 Gif sur Yvette, France

(Reçu le 13 avril 1978, révisé le 17 juillet 1978, accepté le 20 juillet 1978)

\begin{abstract}
Résumé. - Cet article présente des résultats concernant la réflexion d'atomes métastables d'hélium d'énergie cinétique thermique par une surface monocristalline de molybdène $/ 110 /$, en fonction de l'angle d'incidence et du taux de couverture d'oxygène. Les mesures sont effectuées avec une méthode de temps de vol. Le coefficient de réflexion, très bas pour les faibles angles d'incidence, est important pour les grands angles et croît avec le taux d'exposition à un environnement contrôlé d'oxygène. La forme des lobes de réflexion est aussi très influencée par ces deux paramètres.
\end{abstract}

\begin{abstract}
This article presents the results of work concerned with the reflection of metastable helium atoms with thermal kinetic energy by a monocrystalline surface of molybdenum $/ 110 /$, as a function of angle of incidence and degree of oxygen coverage. Measurements were taken with a time of flight method. The reflection coefficient, very low with small angles of incidence, is greater with high angles, and increases with the rate of exposure to a controlled oxygen atmosphere. The form of the reflection lobes is also strongly influenced by these two parameters.
\end{abstract}

1. Introduction. - The theoretical model developed by Hagstrum [1,2] implies that the interaction of rare gas metastable atoms with a metallic surface can provide information comparable to that supplied by INS concerning the electronic properties of the surface.

Until recently, research essentially dealt with measurement of the electronic yield of the interaction, and related it to the nature of the surface and to its chemical state $[3,4,5,6,7]$. Only recently did the first results appear, obtained by secondary electron spectroscopy [8]. In every case, the authors had to overcome difficulties, both experimental and theoretical. In the incident beam, it is not only the metastable atoms which are capable of causing secondary electronic emission. This can be done by :

- UV photons (21.2 eV and $40.6 \mathrm{eV}$ for helium),

- atoms excited to Rydberg levels [9],

- atoms which are neutral at the fundamental level but which have high kinetic energy [10].

The only way to overcome these problems and eliminate the effect of photons and fast neutral atoms is to use a time of flight method. As for the strongly excited atoms, we have shown [9] that an electrical field of $2 \mathrm{kV} / \mathrm{cm}$ suffices to eliminate them by the Stark effect.
Furthermore, as shown by Hagstrum, de-excitation can proceed by two means : resonant ionization followed by neutralization, or direct de-excitation. It should be added that, in principle, it is not possible to exclude the possibility that some atoms undergo radiative de-excitation by the broadening of the metastable level during their interaction with the surface [11]. The interpretation of the results thus requires reliance on certain assumptions, suggested by the results or by the experimental conditions.

Since not all incident atoms are de-excited, but a portion of them is reflected [12], with this reflection occurring in a variable manner according to the angle of incidence and the degree of covering of the surface by impurities, it appears necessary, in the planned analytical conditions, to make the most complete possible review of metastable/surface interaction. This concern led to the investigation presented here, which was intended to determine the variation in the reflection coefficient of metastable helium from a molybdenum /110/ target, as a function of angle of incidence and the rate of exposire to a controlled oxygen atmosphere.

2. Measurement apparatus and method. - An experimental assembly designed to investigate metastable/surface interaction must, in addition to the 
usual characteristics of analytical systems for surface physics, possess the means necessary for measuring the angular distribution of reflected and secondary particles, and for the energy analysis of the secondary electrons. The experimental assembly used here and shown in figure 1 supplements the system described elsewhere [7, 9] and satisfies these considerations.

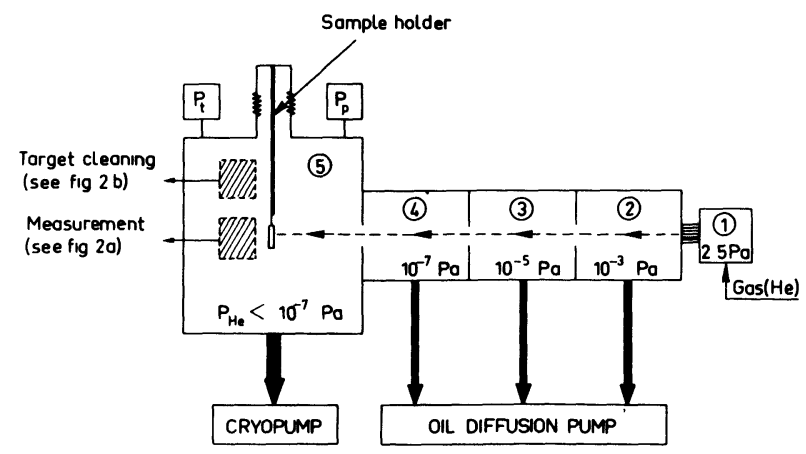

FIG. 1. - Schematic view of the experimental arrangement.

2.1 SourCE. - The atom beam, obtained by the diffusion of atoms through a microchannel plate (length/diameter ratio 50), is excited by electron bombardment. In order to separate, by time of flight, the effects of the various particles resulting from this bombardment; the electron emission is pulsed in synchronization with the scanning of a multichannel analyzer.

This pulsing process has one drawback : excitation transfers are possible, in the beam, between metastable atoms and atoms at the fundamental level. Hence the velocity distribution of the metastable atoms varies along the beam, and only the distribution of arrival times in the vicinity of the target can be known with certainty. We nevertheless preferred this pulsing technique to the use of a mechanical chopper, due to its great operating flexibility, especially with a bakeable system.

For the experiments whose results are shown here, the excitation duration per cycle, the density of the atom jet and the electronic intensity were adjusted so that the signal was always lower than $10^{4}$ counts per second, because the Channeltron used stops being linear beyond this limit.

2.2 EXPERIMENTAL CHAMBER (Fig. 2). - At the center of the chamber, the circular target (diameter $12 \mathrm{~mm}$, thickness $1 \mathrm{~mm}$ ) is suspended from an $x y z$ sample holder, featuring rotation about the vertical axis and rotation about the horizontal axis of the target. Movement along the vertical axis $z z^{\prime}$ allows the target to be brought facing the atom beam (measurement plane) or to a higher level (cleaning plane).

Also in the measurement plane is a mobile support rotating around the target. Attached to this support are a Channeltron for measurements of total flux in a given direction (incident flux or secondary flux after

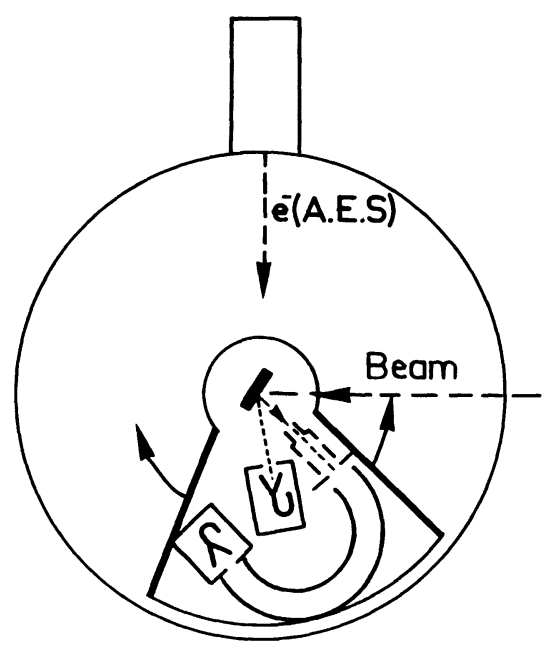

a)

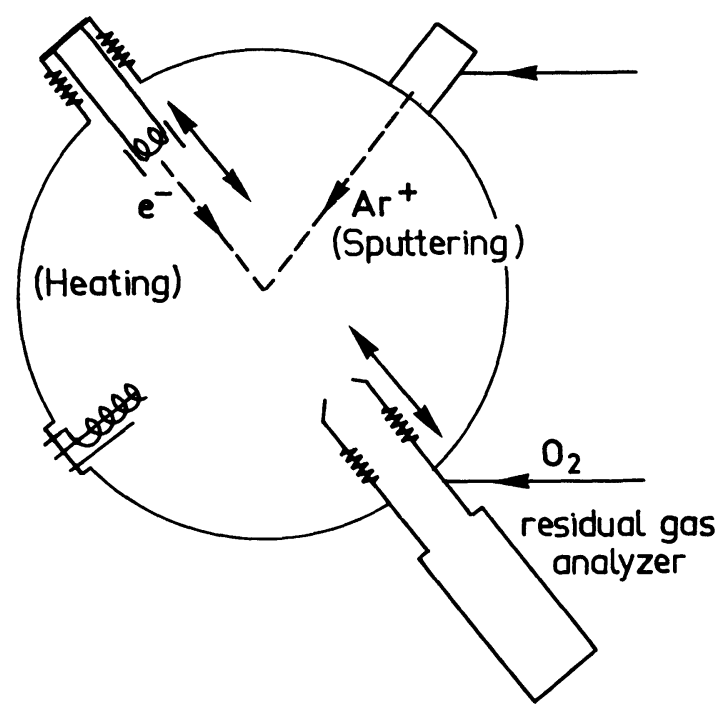

b)

FIG. 2. - Experimental chamber $(2 a)$ : measurement plane ; $(2 b)$ : cleaning plane.

interaction with the target), and an electrostatic analyzer with cylindrical optics [13] for energy analysis of the charged secondary particles emitted in the direction of the axis of its input optics. Stationary in the same plane is an electron gun $(0$ to $3 \mathrm{kV})$ which, combined with the electrostatic analyzer, is intended for characterization of the target surface by AES.

Electron and ion guns are placed in the cleaning plane (cleaning by heating and by sputtering), as well as a cell intended for characterization of the surface by pulsed thermal desorption. This cell can be positioned so that its slit is obturated by the target. Thus, in spite of the high pumping speed into the measuring vessel, thermal desorption peaks are high enough to be analyzed by quadrupole filter. This cell also serves to expose the target to known quantities of gas. 
Owing to the presence of differential pumping stages between the source and the experimental chamber, and to the high pumping rate permitted by the use of a cryopump, after baking at $250^{\circ} \mathrm{C}$, the basic pressure in the experimental chamber is lower than $5 \times 10^{-9} \mathrm{~Pa}$ and remains lower than $10^{-7} \mathrm{~Pa}$ in the presence of a helium atom beam, which section is $8 \mathrm{~mm}$ large on the target axis.

Note also that one of the volumes corresponding to the differential pumping zones is used for the destruction of the Rydberg states by the Stark effect between two electrodes. Furthermore, checking with an intense magnetic field shows that the beam only contains neutral particles.

2.3 Measurement METHOD. - The reflection of metastable helium was investigated by using a Channeltron placed on the mobile support, and polarized so that the secondary electrons emitted from the target could not be collected. We have explained above that the operating parameters of the source were set at a level such that the Channeltron, even if placed in direct view, did not receive a flux greater than $10^{4}$ particles per second.

Zone by zone integration of the signal accumulated in the multichannel analyzer allows analysis of the influence of interaction conditions on the reflection of these particles (intensity and direction). The accumulation time for each point is such that the construction of reflection lobes always takes less than one hour. Owing to the partial pressure of the chemisorbable gases in the neighbourhood of the target $\left(<5 \times 10^{-9} \mathrm{~Pa}\right)$, the rate of impurity coverage of the target only varies within negligible proportions during measurement.

3. Results and discussion. - 3.1 FORM OF REFLECTION LOBES. - For information purposes, figure 3 shows the results obtained for different angles of incidence and different total exposures to oxygen.

Remarkable directivity of the reflected atoms can be observed, far greater than the level observed by Craig and Dickinson [12] with a germanium target. However, while intensity increases when the target is exposed to oxygen, the directivity of reflection diminishes; the lobe broadens when adsorption reaches a few tens of Langmuirs. This observation can be interpreted as the passage from one state in which the energy exchange between the incident particle and the solid occurs by means of a collision of metastable atoms with metallic atoms (or strongly bound adsorbed atoms), to another state in which the collision occurs between metastable atoms and weakly bound adsorbed atoms. One know in fact, that form of reflection lobes has something to do with coupling between surface atom knocked by incident atom and the other network atoms.

So that, the possibility emerges of investigating the adsorption of gases on solids in conditions which are
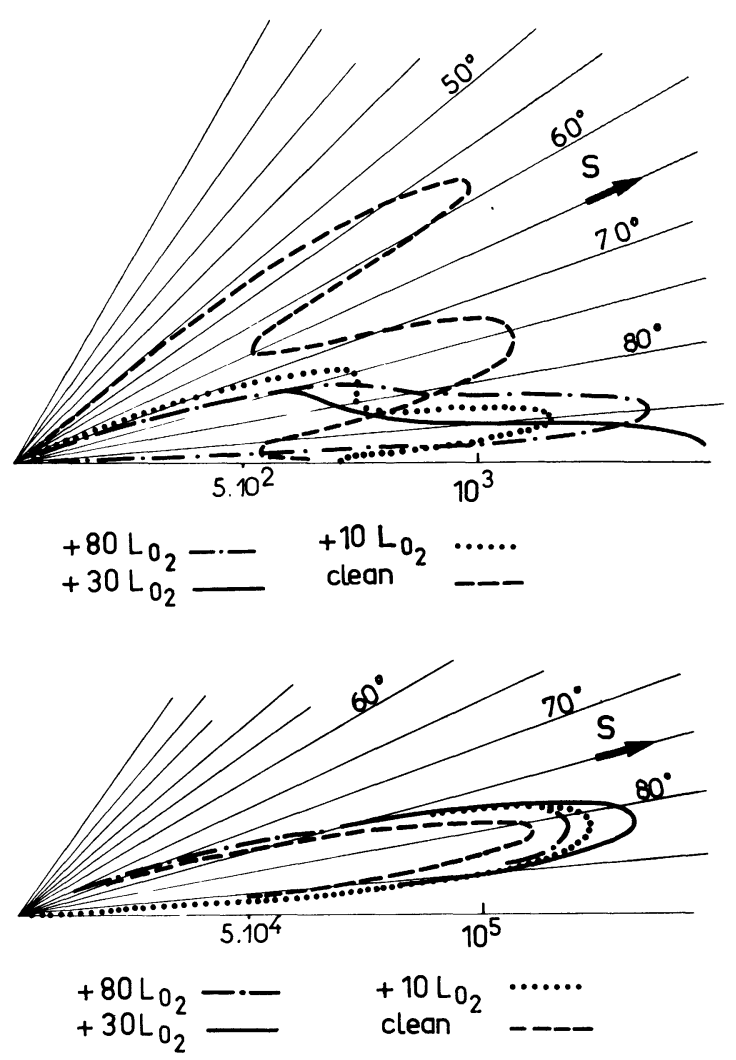

FIG. 3. - Angular distribution of reflected metastable atoms. $\mathrm{S}$ is the specular direction, and the number is the reflected atoms quantity. Experimental data are set from 5 to 5 degrees. Incertainties which are not drawn on the curves, are respectively inferior to $5 \%$ for 65 degrees of incidence and $5 \%$ for 75 degrees of incidence.

difficult to obtain experimentally by nondestructive processes.

3.2 VARIATION IN REFLECTION COEFFICIENT. - As suggested by figure 3 , the reflection coefficient of $\mathrm{He}^{*}$ on Mo $/ 110 /$ is very sensitive to the angle of incidence (Fig. 4) and to oxygen adsorption (Fig. 5).

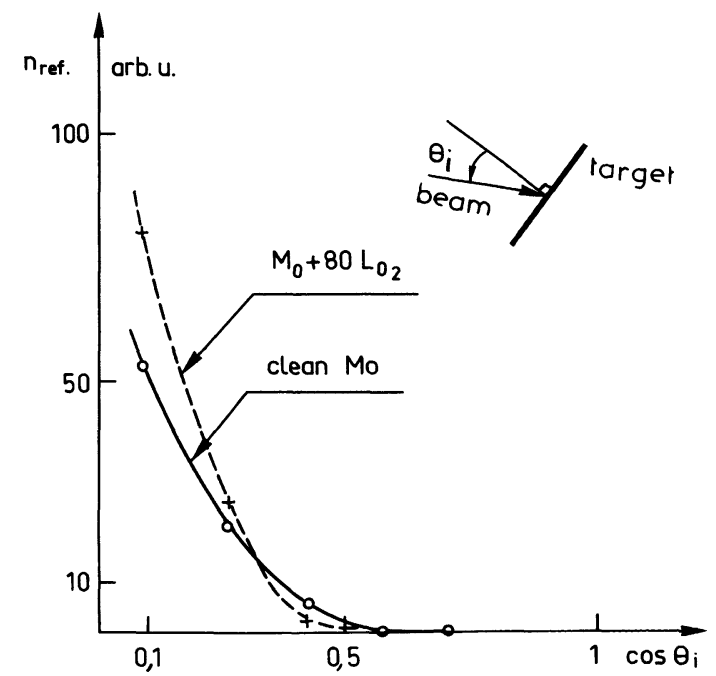

FIG. 4. - Variation of the number of reflected atoms as a function of incidence angle. 


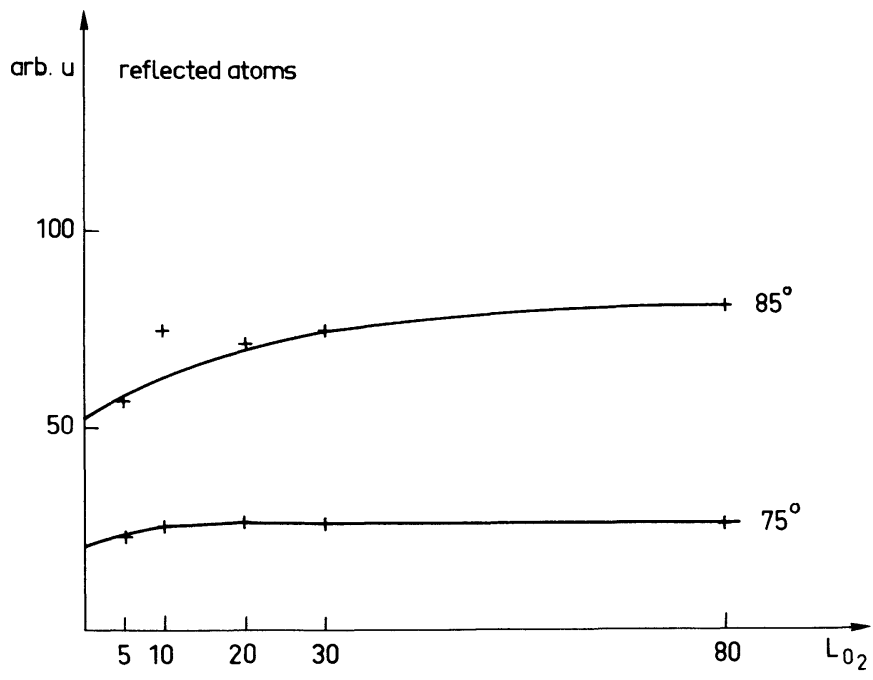

FIG. 5. - Variation of the number of reflected atoms versus the rate of exposure to oxygen atmosphere.

As observed by Oliphant [14] and Craig and Dickinson [12], the decrease in the reflection coefficient with angle of incidence is confirmed by our results; the reflected atoms, which are practically negligible with small angles, become measurable around 55 degrees and become significant with nearly grazing incidence. This implies that the probability of de-excitation of the metastable atom is directly related to the component of the velocity of the incident atom which is normal to the surface. On the other hand, basing on a direct de-excitation model, Colas and Lamb [15] calculated a significative decreasing of the probability of de-excitation of the metastable atom, when incident angle increases.

As for the influence of oxygen adsorption on the number of reflected atoms, we are inclined to compare this with the results obtained by Denninger [16] using isochromatic spectroscopy; the density of the free states of molybdenum decreases with oxygen adsorp- tion up to an exposure of about 20 Langmuirs. Thus, the increase in the reflection coefficient is the consequence of the lower probability of resonant ionization of the metastable atoms (tunnel transition of the electron excited to the free states of the solid).

It results from the foregoing discussion that the probability of direct de-excitation of the metastable atom is insufficient to compensate a decrease in the probability of the de-excitation process by ionization. This situation can easily be explained because, in the case at hand, the adsorbed oxygen forms a screen between the metastable atom and the solid, and consequently prevents the hole created by the fundamental level of the atom from being filled in by an Auger type process with the electrons of the solid, the probability of this Auger process with the electrons of the oxygen being relatively lower.

4. Conclusions. - The investigation of the reflection of metastable helium atoms on a monocrystalline molybdenum surface is a significant contribution to the analysis of the net results of the interaction; the number of atoms reflected in the metastable state is negligible with angles of incidence smaller than 50 degrees, and only becomes significant with nearly grazing incidence.

Furthermore, it confirms the hypothesis postulated by Hagstrum and confirmed experimentally [9] : for a metal of which the work-function is greater than the ionization potential of the metastable atom, the latter is de-excited by resonant ionization, and the ion is then neutralized by an Auger process.

Acknowledgments. - The authors wish to stress the decisive role played by Mr. R. Barré, who directed the mechanical design of the experimental system and ensured its maintenance, and they wish to thank Mrs J. J. Le Grand and A. Schmouchkovitch [17, 18], who contributed actively to the development of the electronic instrumentation.

\section{References}

[1] Hagstrum, H. D., Phys. Rev. 96 (1954) 336.

[2] Hagstrum, H. D., Takeishi, T., Pretzer, D. D., Phys. Rev. 130 (1965) A 256.

[3] Delchar, T. A., Mac Lennan, D. A., Landers, A. M., J. Chem. Phys. 50 (1969) 1779.

[4] Dunning, F. B., Smith, A. C., Stebbings, R. F., J. Phys. B 4 (1971) 1683

[5] Allison, W., Dunning, F. B., Smith, A. C. H., J. Phys. B 5 (1972) 1175.

[6] Mac Lennan, D. A., Delchar, T. A., J. Chem. Phys. 50 (1969) 1772.

[7] Boiziau, C., Dose, V., Roussel, J., Surf. Sci. 61 (1976) 412.

[8] Johnson, P. D., Delchar, T. A., J. Phys. E 10 (1977) 428.

[9] Roussel, J., Boiziau, C., J. Physique 38 (1977) 757
[10] Roussel, J., Boiziau, C., Cong. Nat. Phys. Plasmas Paris 6-10 déc. 1976

[11] Nowak, G., Borst, W. L., Fricke, J., to be publis. in Phys. Rev. A.

[12] Craig, J. H., Dickinson, J. T., J. Vac. Sci. Technol. 10 (1973) 319.

[13] Ballu, Y., J. Microsc. Spectr. Electr. 2 (1977) 231.

[14] Oliphant, M. L. E., Proc. R. Soc. A 124 (1929) 228.

[15] Cobas, A., Lamb, W. E., Phys. Rev. 65 (1944) 327.

[16] Denninger, G. A., Dipl. Arbeit, Universität Würzburg, RFA, 1978.

[17] Le Grand, J. J., Sмmouchkovitch, A., Autom. Infor. Ind. $\mathbf{5 4}$ (1977) 17.

[18] Le Grand, J. J., Autom. Infor. Ind. 58 (1977) 67. 